DEC 271990

\title{
LASER ULTRASONICS: \\ CURRENT RESEARCH NEEDS
}

September 26, 1990

James W. Wagner

Center for Nondestructive Evaluation

The Johns Hopkins University

Baltimore, Maryland 21218

A Final Report of a Program Entitled "Laser-Ultrasonics"

(August 1, 1990 - September 30, 1990)

Submitted to Lawrence Livermore National Laboratories 


\section{DISOINIMIER}

Work performed under the auspices of the C..t. Department of tinergy hy Lawrence Livermore National Iaboratery under contrace number $11-7405 .+\mathrm{NG} ;-48$.

This document was prepared as an accoumt of work sponsored by an ageney of the Inited Sitates (jovernment. Veither the I inited States Gover sment nor the I 'nisersity of California nor any of their cimployec's, makes any warranty. cxpress or implied, or assumes any lejal liability or responsibility for the accuracy, completeness, or usefuliness of any information, apparatus, product, or process disclessed, or represents that its use would mot infringe privately owned rights. Reference herein to any specific commercial prodacts, precess, or service bs trade name, trademark, manufacturer, or otherwiste, does not necessarily constitute or imply its condorsement, recommendation, or favoring by the Linited States coocermenent or the Iniversity of California. The visus and opinions of autloors espressed herein do not necerssarily state or reflect these of the I nited States Goternument or the liniversity of Californial, and shatl not be used fur advertising or product endorsement purposes. 
Introduction

Laser-ultrasonics refers to a range of technologies involving the use of laser electrooptical systems both to generate and to detect ultrasonic signals in and on materials and structures. Such systems have been developed to permit classical ultrasonic measurements for materials characterization and defect identification and measurement. Unlike conventional piezoelectric ultrasonic transducers which require mechanical contact with the object being inspected, laser-ultrasonic sytems are non-contacting and remote. The capability for remote measurement arises from the fact that the transduction from light energy to acoustic energy, and in turn from acoustic energy into optical information, takes place as a result of direct physical interaction between the propagating laser light, used to generate or detect ultrasound, and the surface of the test object. No intermediate transduction or couplant is required. This capability for making remote measurements is an advantage over other noncontacting ultrasonic transducers, such as capacitance transducers and electro-magnetic acoustic transducers (EMATs) where relatively low frequency and short range electo-magnetic fields are employed. From the point of view of one concerned with practical applicatations of ultrasonic inspection and measurment methods, laser-ultrasonic systems offer the flexibility which, in principle, should permit remote ultrasonic measurements to be performed on objects at elevated temperatures or in hostile environments. Furthermore, rapid scanning of even complex object shapes should be facilitated since there is no need for direct mechanical coupling. Also from the point of view of those less interested in immediate applications but instead are more concerned with the fundamental aspects of ultrasonics, laser-ultrasonic systerns offer great advantages. Laser-ultrasonic systems can be designed and constructed with ex'remely wide and flat 
detection bandwidth so that ultrasonic vibrational displacements can be recorded with high fidelity. In addition, there is no mechanical loading of the surface to damp, absorb, or otherwise distort the propogating acoustic energy. This feature has been used to great advantage in performing ultrasonic measurements in thin plates and films.

In spite of the great advantages offered by laser-ultrasonics, there are severe limitations which restrict its application. In fact, based upon the performance of current state-of-the-art laser-ultrasonic systems, it is almost always more advantageous to use conventional ultrasonic tranduction methods, if possible for a given application, than it is to apply laser-ultrasonics. In short, the main reason leading to this conclusion is the poor system detection sensitivity of laser-ultrasonic systems compared with piezoelectric transducer systems. (The theoretical and practical reasons limiting laser-ultrasonic detection sensitivity will be discussed shortly.) The ramefications of this limited sensitivity are many. With limited detection sensitivity, poor signal-to-noise ratio (SNR) signals from the laserultrasonic system result in a corresponding decrease in the precision with which ultrasonic attenuation and velociiy measurements can be made. As the required ultrasonic propagation distance or material attenuation increases, SNR may fall so low that no signal may be detected at all.

From the discussion above, it is clear that laser based methods for generation and detection of ultrasound are well established for laboratory use. However, for use in the field as a nondestructive testing tool or in the factory as a sensor for process control, laser ultrasonic methods suffer by comparison with more conventional contact transducer techniques. In order to overcome the linitations which restrict the application of laser 
ultrasonic methods, several research needs have been identified. By addressing these needs, it may be possible to broaden the range of application for laser ultrasonics.

\section{Sensitivity}

The lack of measurement sensitivity relative to contact transducer methods is, perhaps, the single most important factor limiting the applicability of laser ultrasonic methods. In fact, most of the research needs presented in this report result directly from the poor overall system detection sensitivity of laser ultrasonics. It is generally the case for virtually all optical methods for detecting ultrasonic vibrations that the noise floor limiting detection sensitivity is established by shot noise associated with the optical detection process. In other words, noise associated with electronic instrumentation or environmental conditions can, in principle, be made small compared with the noise associated with the detection of light reflected from the surface of a test object into a laser ultrasonic detection system. For a detection system whose sensitivity is shot noise limited, the signal-to-noise ratio varies as a function of surface displacement, $\delta$, optical power on the detector, $\mathrm{P}$, and overall system bandwidth, B, according to the relation given below.

$$
S N R \propto \frac{P}{B} \times 8^{2} \quad \text { Equation } 1
$$

Therefore, the degree to which electro-optic technology will allow, and for a particular testing or measurement application, it is possible to affect the sensitivity of laser ultrasonic methods by controlling the three parameters in this expression. 


\section{A. Detector Power, P}

It stands to reason that since the source of shot noise comes from the conversion process of optical to electrical power that it should be related to the amount of optical power falling on a photodetector such as a photodiode or photomultiplier detector. In fact, the shot noise power generated by a system is related directly to the rate at which individual photons fall on the sensitive area of an optical detector. With increasing intensity, the number of photons per seconds falling on the detector increases as does the associated shot noise power. Fortunately, the signal power, that portion of the light intensity which carries with it the information about the vibration or displacement of a surface, increases as the square of the optical power falling on the detector. Since the noise power increases directly with light intensity and since the signal power increases with the square of light intensity, there is a net improvement in the ratio of signal power to noise power as the amount of light is increased in an optical laser ultrasonic detection system such as an interferometer.

This need to maximize the light throughput in an optical interferometer system for ultrasonic detection explains why most of the successful applications of laser ultrasonic systems are performed in laboratories where the surface of test specimens can be polished to give a high specular reflectivity. Under such controlled conditions, sensitive detection of ultrasonic signals can be performed using helium neon laser sources with output powers below 5 milliwatts. In the more general case where objects to be tested may have rough and scattering surface finishes, the amount of light ultimately coupled back into a detection interferometer system may be so small that only very large surface displacem nts or extremely narrowband signals may be detected reliably. Nevertheless, there exist applications such as in the microelectronics industry, for example, where the surface of 
objects and devices to be inspected may have well-polished surfaces as a direct consequence of the device manufacturing process.

For the more general case where test objects may have optically rough surfaces, several schemes are being investigated. Some of these schemes have been sufficiently successful to have been reduced to practice in very specific testing applications. In every case, they involve the use of higher power lasers in the ultrasonic detection system. Clearly, if the surface of an object scatters light so that the intensity which ultimately reaches a photodetector is reduced, it makes sense that a higher power laser source should permit a greater quantity of light to be collected. In using larger lasers in the detection systems, however, one must be careful to insure that the laser noise remains below the detection system shot notice in order that the system remain shot-noise limited. It is for this reason that larger helium neon lasers are not, in general, considered as candidates for higher power interferometer systems. Instead, etalon stabilized argon ion lasers and even very specially stabilized long-pulsed duration, seeded Nd:YAG lasers have been employed with good success (but at comparatively high costs). As an example, a modified long-pulsed Nd:YAG laser system reportedly has been incorporated into a laser ultrasonic system to measure wall thickness in hot steel pipe extrusions. During its output pulse, optical power in excess of 1000 watts is delivered to the surface so that sufficient light can be scattered back into the interferometer to allow sensitive detection of ultrasonic displacements.

In addition to increasing the amount of light reaching the photodetector of an interferometer system, it is necessary that the interferometer performance not be compromised by any spatial structure, such as speckle, in the reflected light. For this reason, when narrow beam interferometer systems are used for ultrasonic detection on 
rough surfaces, the beams from the interferometer are focused to a diffraction limited spot. This insures that whatever light is reflected back in the direction of the interferometer will be imaged as a single, speckle-free beam when it arrives at the photodetector. While this approach works quite successfully, it requires that the stand-off distance between the interferometer and the object surface be very carefully controlled. Fabry-Perot and delaytype interferometers, however, can be operated with large collection apertures so that an entire speckle field, and not just a single speckle, can be combined interferometrically to permit sensitive detection of ultrasonic surface displacements.

Finally, a method still in the research laboratories is one which incorporates the use of phase conjugate optical devices which operate as mirrors of sorts to reflect the conjugate or "time-reversed" replica of a collected speckle field back onto the object surface. When this conjugate beam encounters the object surface, it is "rescattered" by the surface back into what should be, ideally, a uniform beam which can be collected and processed by any of the classical interferometer methods. In theory, there is an added advantage to the phase conjugate assisted interferometric methods in that the reflectivity of a phase conjugate mirror can be made to exceed $100 \%$ ! Consequently, absorption losses at the material surface may be compensated. Other experimental possibilities for increasing detector power remain to be explored, including the possibility of incorporating longer wavelength optical sources which would have less sensitivity to surface roughness.

\section{B. Bandwidth, B}

From the proportionality presented earlier, it would appear that a reduction in the electronic signal bandwidth of an interferometer detection system will, in general, increase 
the signal-to-noise ratio. This can be understood on an intuitive level by recognizing that the nature of shot noise power is that it has a uniform energy density over the entire frequency spectrum and as such, falls into the category of white noise. Clearly then, with increasing system bandwidth, greater amounts of shot noise power are added to the signal of interest. Conversely, by reducing system bandwidth, the shot noise power is correspondingly reduced.

Unfortunaiely, when a single pulse high energy laser is used to generate the acoustic signal of interest, the energy in the generated acoustic signal may extend over a very broad range of frequencies as well. Therefore, in order to capture and reproduce an accurate analogue of the ultrasonic signal, it is necessary to maintain a broad bandwidth in the interferometric detector system. As a consequence, one may narrow the bandwidth of the detection system to eliminate shot noise only up to the point that further bandwidth reduction begins to eliminate signal power as well. Once that point is reached, no further enhancement of the overall signal-to-noise ratio can be achieved by further bandwidth reduction.

Investigators at the Johns Hopkins University have, for the past several years, investigated means to modify lasers used as ultrasonic sources in order that narrow band tone burst-like acoustic signals can be generated. Multiple Q-switching, repetitive cavity dumping, and, most recently, long cavity mode locked solid state laser sy ms have been designed and tested. The latter system causes a sequence of optical pulses to be emitted from the laser for each single pulse of the flash lamps. The repetition frequency of the pulses within each burst can be varied from below $2.5 \mathrm{MHz}$ to about $160 \mathrm{MHz}$. The spectral distribution of the ultrasonic signals generatrd by the tone burst output of this laser 
is greatly reduced in bandwidth, thereby permitting a commensurate reduction in signal bandwidth of the optical detector system so that overall, laser ultrasonic system signal-tonoise and sensitivity, is increased.

\section{C. $\quad$ Surface Displacement, $\delta$}

Improvements in overall laser ultrasonic system sensitivity can be affected most directly by increasing the surface displacements associated with the ultrasonic signal propagating in the object under inspection. Means for doing so can be understood by recalling the primary mechanisms by which a high energy laser beam can generate sound at the surface of an absorbing object. When the power densities of the beam at the object surface are sufficiently high, ablation of the object material may occur so that the momentum of the mass leaving the surface contributes directly to a compressive force normal to the object surface. This is the most efficient source for generating longitudinal waves through the bulk of the object. Unforiunately, there is associated damage to the object surface which may not be acceptable for many applications. However, in early stages of materials processing where the surface will be machined or otherwise modified in subsequent processing steps, the small amount of damage imparted by the laser beam may be insignificant. A non-damaging method related to direct material ablation is one in which the surface is coated with a material which may be a strong laser absorber but is itself ablated or evaporated rapidly, thus still contributing to strong elastic wave generation but protecting the surface of the object itself.

A second mode of laser generation of ultrasound is that in which the laser power density is below that sufficient to cause material ablation. The source for elastic waves in 
this case results from the rapid heating of a small volume of the object material right at the surface causing a rapid thermomechanical expansion which in turn generates propagating acoustic waves. It is this thermoelastic regime of laser ultrasonic generation which is truly nondestructive and noncontacting. From a small point on the object surface, bulk, longitudinal, and shear waves, as well as Rayleigh surface waves, are generated by the thermoelastic source. There exists an inherent directivity of the energy which propagates from this source, however, so that both shear and longitudinal waves propagate most strongly in off-epicentral direction.

An intermediate regime for laser ultrasonic detection is one in which the object surface is mechanically constrained by a transparent material through which laser powers below the ablative threshold can be transmitted and absorbed by the object. Expansion of the object surface is now constrained by the coating material so that the on-epicenter directivity of longitudinal wave components are enhanced.

With this understanding of the nature of laser sources, certain principles become clear which should permit one to configure a laser ultrasonic system to take advantage of the greatest displacements produced by the laser sources. For example, if a testing application is restricted such that thermoelastic sources alone may be used, one can expect greatest longitudinal and shear wave displacements at points off-epicenter and should configure the test setup so that the receiving point is appropriately positioned relative to the generating point. For example, in aluminum and its alloys, the longitudinal wave propagates with greatest amplitude at an angle of about $65^{\circ}$ off-epicenter, shear waves at about $30^{\circ}$. Given the thickness of the aluminum being tested, one can predict the location of peak 
surfare displacements on the backside of the specimen resulting from these propagating wave modes.

As mentioned before, in applications where small amounts of surface damage can be tolerated, a larger acoustic signal may be generated by increasing laser power density into the ablative regime. Depending on the material, a plasma shielding effect may reduce the efficiency with which strong epicentral displacements can be generated as laser power density increases. Under such conditions, increased surface displacements still can be obtained by increasing the total laser power but delivering it over a sufficiently broad area of the specimen that local power densities do not exceed the threshold beyond which plasma shielding is observed.

Increasing the amount of acoustic energy coupled into the bulk of a test object can also be accomplished by using a "packet" of repetitive laser pulses with peak power densities below that which may cause material ablation but having a combined energy greater than or equal to that which would have caused ablation if delivered in a single pulse. The resulting acoustic waveform in this case will no longer be a single spike but instead a train of pulses. This pulse train or tone burst energy is such that the elastic energy is grouped over a narrower range of frequencies so that narrow band interferometry systems can be used for detection.

Lastly, arrays of generating points may be used to produce ultrasonic signals where the directivity of certain features of the elastic waves can be controlled. By appropriately controlling the time delay between a sequence of laser pulses which illuminate a series of array points on the surface of the object, the angle corresponding to the maximum particle displacement can be controlled. Furthermore, it has been demonstrated that if an array of 
closely spaced lines is illuminated simultaneously with a single laser pulse, a tone burst of ultrasonic energy may be detected off-axis at an angle depencient upon the separation of lines in the array. In this 'atter case, there is once again the advantage that a narrow band receiver can be used. Lnfortunately, it is too often the case, even among investigators in the field, that the use and effect of patterned illumination is drawn too closely in parallel with RF-phased antenna arrays. In the case of laser generation of ultrasound, particle displacements associated with propagating bulk modes are monophasic for a given propagation direction. While this permits reinforcement of the displacement waveforms with appropriate spacing and phring of the array, there is, unfortunately, no corresponding destructive interference in other directions in the bulk material. Consequently, only amplitude features, such as pulse height or fundamental tone burst frequency, can be directed by altering the array parameters. That being the case, the total energy directivity from a laser array is unaltered from the energy directivity that would arise from a uniform pattern illuminating the object over the same footprint as that of the phased array pattern. Nevertheless, there may be some advantage and enhancement in overall system sensitivity to be obtained using phased laser array.

\section{Laser Ultrasonic Source Physics and Modeling}

Existing models predict quite accurately the displacements which one might expect

to see as a result of propagation of laser generated ultrasonic sources in bulk materials. With only a couple of exceptions, these models are applied in two stages of reasoning. First, the effect of laser heating or ablation on the surtace of the material is modeled so that a mechanical equivalent is derived. In the second stage, then, this mechanical equivalent ior 
the laser source is used as a forcing function from which a Green's function-inke solution for elastic propagation in the material is obtained. Again. this approach is quite adequate when dealing with most bulk materials. In some cases, however, the decoupling between the thermal and mechanical effects on the material which takes place as a result of the twostage models fails to predict accurately the ultrasonic displacement waveforms which are observed in thermally anisotropic materials or in extremely thin films. It will be important to continue to develop more unified theories of laser ultrasonic generation in materials so that thermally anisotropic materials (e.g., composites), and so that thin film materials, such as those used in coatings and in the microelectronics industry, can be inspected using laser ultrasonic methods.

\section{Conclusions}

While laser ultrasonic methods have shown demonstrated promise in laboratory applications, there remain gaps in our understanding of how these methods might apply to new and more complex materials. Furthermore, a great variety of testing applications could be addressed by laser ultrasonic methods as efforts succeed in improving the overall measurement sensitivity of this remote and potentially nondestructive testing modality. 


\section{Topical Bibliography}

This bibliography is arranged by topic with a small list of key papers presented in each topical category. In most cases, review articles are listed which, in turn, cite papers of narrower scope which provide details on specific aspects of laser-ultrasonics.

\section{Laser Generation of Ultrasound}

C.B. Scruby, R.J. Dewhurst, D.A. Hutchins, and S.B. Palmer, Laser Generation of Ultrasound in Metals, in Research Techniques in Nondestructive Testing, Vol. 5 (R.S. Sharpe, ed.), Academic Press, New York, 281-327, (1982).

D.A. Hutchins, Ultrasonic Generation by Pulsed Lasers, Physical Acoustics, Vol. 18, 21-123, (1988).

\section{Laser Detection of Ultrasound}

J.-P. Monchalin, Optical Detection of Ultrasound, IEEE Trans. UFFC-33(5), 485-499, (1986).

J.W. Wagner, Optical Detection of Ultrasound, Physical Acoustics, Vol. 19, 201-266, (1990).

\section{Analytical/Numerical Modeling}

R.M. White, Generation of Elastic Waves by Transient Surface Heating, J. Appl. Phys., 34(12) 3559-3567, (1963).

L.R.F. Rose, Point-Source Representation for Laser-Generated Ultrasound, J. Acoust. Soc. Am., 75(3), 723-732, (1984).

U. Schleichert, K.J. Langenberg, W. Arnold, and S. Fasbender, A Quantitative Theory of Laser-Generated Ultrasound, in Review of Progress in Quantitative Nondestructive Evaluation, (D.O. Thompson, D.E. Chimenti, eds.), Plenum Press, New York, Vol. 8A, 489-496, (1989). 
F.A. McDonald, A New Method for Calculation of Laser-Generated Ultrasonic Pulses, in Review of Progress in Ouantitative Nondestructive Evaluation, (D.O. Thompson, D.E. Chimenti, eds.), Plenum Press, New York, Vol. 9A, 503-510, (1990).

J.B. Spicer, A.D.W. McKie, and J.W. Wagner, Quantitative Theory for Laser Ultrasonic Waves in a Thin Plate, Appl. Phys. Lett., 57(18) to be published 29 October, 1990. 

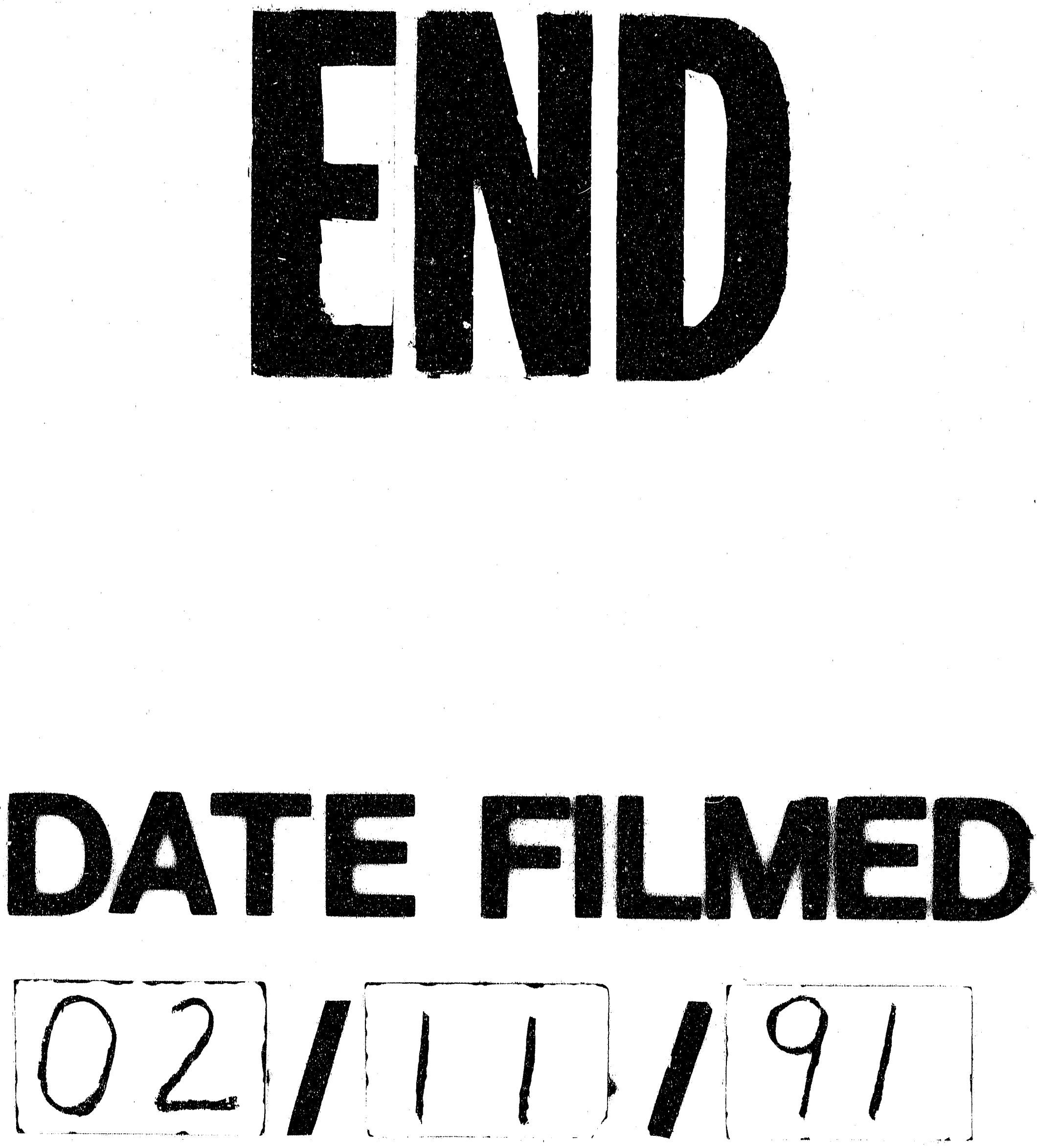
